\title{
Tractography-Guided Stimulation of Somatosensory Fibers for Thalamic Pain Relief
}

\author{
Stefan Hunsche ${ }^{a}$ Dieter Sauner ${ }^{b}$ Matthias J.R. Runge ${ }^{a}$ Doris Lenartz ${ }^{a}$ \\ Faycal El Majdoub ${ }^{a}$ Harald Treuer ${ }^{a}$ Volker Sturm ${ }^{a}$ Mohammad Maarouf ${ }^{a}$ \\ a Department of Stereotactic and Functional Neurosurgery, University of Cologne, Cologne, and ${ }^{b}$ Department of \\ Radiology, Fachkrankenhaus Hubertusburg, St. Georg Corporation Leipzig, Wermsdorf, Germany
}

\section{Key Words}

Stereotactic surgery . Deep brain stimulation · Diffusion tensor imaging $\cdot$ Fiber tracking $\cdot$ Thalamic pain · Internal capsule

\begin{abstract}
Background: The spinothalamocortical tract (STC) is seen as a neural tract responsible for or involved in the generation or transmission of thalamic pain. Either the thalamus itself or the posterior limb of the internal capsule (PLIC) are targets for deep brain stimulation (DBS) in patients with thalamic pain, but due to its low contrast, conventional MRI cannot visualize the STC directly. Objectives: To show the feasibility of integrating diffusion tensor imaging-based tractography into the stereotactic treatment planning for identification of an object-oriented lead trajectory that allows STC-DBS with multiple electrode contacts. Methods: Diffusion tensor imaging was performed in 4 patients with thalamic pain. The STC was modeled and integrated into the stereotactic treatment planning for DBS. DBS-lead implantation was done according to trajectory planning along the modeled STC at the level of the PLIC. Results: After implantation, electrode stimulation was possible over a length of more than $20 \mathrm{~mm}$ with a tractography-based trajectory along the PLIC part of the STC. After a follow-up of 12 months, pain relief of more than $40 \%$ was achieved in 3 of 4 patients with rating on a visual
\end{abstract}

\section{KARGER}

(C) 2013 S. Karger AG, Base

1011-6125/13/0915-0328\$38.00/0

E-Mail karger@karger.com

www.karger.com/sfn analogue scale. In 1 patient, stimulation failed to reach any long-lasting positive effects. Conclusions: Integrating tractography data into stereotactic planning of DBS in thalamic pain is technically feasible. It can be used to identify a lead trajectory that allows for multiple contact stimulation along the STC at the level of the PLIC. Due to long-lasting positive stimulation effect, tractography-guided stimulation of sensory fibers seems to be beneficial for thalamic pain relief.

Copyright $\odot 2013$ S. Karger AG, Basel

\section{Introduction}

Thalamic pain as a result of a thalamic lesion, e.g. lacunar stroke, is often unresponsive to pharmacotherapy and can be debilitating [1]. Alteration of the functionality or connectivity of the spinothalamocortical tract (STC) is discussed as part of its pathogenesis [2,3]. Successful pain relief has been reported by deep brain stimulation (DBS) of either the thalamus itself or the posterior limb of the

Stefan Hunsche and Dieter Sauner contributed equally to this work.

Stefan Hunsche, PhD

Department of Stereotactic and Functional Neurosurgery

Kerpener Strasse 62

DE-50937 Cologne (Germany)

E-Mail stefan.hunsche@uk-koeln.de

Dieter Sauner, MD

Department of Radiology

Fachkrankenhaus Hubertusburg

DE-04779 Wermsdorf (Germany)

E-Mail dieter.fedders@kh-hubertusburg.de 
internal capsule (PLIC) [4-7], an area in which the STC is located on its way from thalamus to postcentral cortex. Alternatively, motor cortex stimulation has been investigated for pain relief $[8,9]$. But motor cortex stimulation, i.e. the covering of the cortical surface with electrodes, has its limits in specific regions, for instance at and in the interhemispheric fissure, a region responsible for the somatotopic representation of the lower limbs. DBS of the PLIC is difficult since usually a single atlas-based target for stimulation is chosen with only a small likelihood for precise targeting and thus successful stimulation.

Our aim was to attain a sufficient visualization of the STC at the level of the PLIC. For that purpose diffusion tensor imaging (DTI) [10] and 'fiber tractography' may turn out as viable tools since technically they enable delineation of white matter tracts [11-15]. Fundamental for these techniques is the fact that diffusion on a molecular level is hindered to a substantially lesser degree along a fiber than perpendicular to it. Sophisticated algorithms enable the mathematical reconstruction of long neural tracts by adding up the directional preferences of several voxels. Reconstruction of specific white matter tracts should allow an objectorientated lead trajectory for intervention planning.

In addition to our aiming for a reliable target selection, our approach to delineate the STC over a long distance is based on the assumption that multiple stimulation sites along the PLIC increase the probability of targeting the STC correctly and/or can increase desirable stimulation effects by multicontact stimulation.

Therefore, the purpose of this paper is to show the feasibility of integrating DTI-based tractography results in stereotactic treatment planning for defining an objectorientated lead trajectory which allows multiple electrode contact stimulation of the STC in patients with thalamic pain.

\section{Patients and Methods}

\section{Patients}

DTI-guided DBS of the somatosensory tract was performed in 4 patients suffering from intractable thalamic pain, with a very likely causal relation between thalamic lesion and pain. Three patients were suffering from poststroke pain and 1 patient from pain developed after brachytherapy-induced remission of a thalamic glioma. Pain affected the whole hemi body in all patients and lasted more than 2 years. Pharmaceutical therapy had been insufficient. Table 1 summarizes the patient data. Preoperation and 3, 6 and 12 months postinterventional pain intensity was assessed with a visual analogue in the range of $1-10$. All patients gave their written informed consent. The institutional review board of the university approved the study.

DTI-Guided Stimulation of Somatosensory Fibers
Table 1. Patient data

\begin{tabular}{|c|c|c|c|c|}
\hline $\begin{array}{l}\text { Patient } \\
\text { No. }\end{array}$ & Sex & Age & Diagnose & Site of pain/characteristic \\
\hline 1 & M & 50 & thalamic infarct & $\begin{array}{l}\text { right hemi body/ } \\
\text { arm accentuated }\end{array}$ \\
\hline 2 & $\mathrm{M}$ & 74 & thalamic infarct & $\begin{array}{l}\text { right hemi body/ } \\
\text { arm accentuated }\end{array}$ \\
\hline 3 & M & 48 & $\begin{array}{l}\text { brachytherapy } \\
\text { induced pain }\end{array}$ & $\begin{array}{l}\text { left hemi body/ } \\
\text { upper limb accentuated }\end{array}$ \\
\hline 4 & $\mathrm{~F}$ & 71 & thalamic infarct & left hemi body \\
\hline
\end{tabular}

\section{MRI and CT Scanning for treatment Planning}

After fixing the patient's head to a stereotactic MRI-safe ceramic frame (MRC, Heidelberg, Germany), a CT scan was performed to estimate the stereotactic transformation from image to frame coordinates. After CT scanning, the patient was moved to a 1.5-tesla intraoperative MRI scanner to get motion artifact-free anatomical images (Intera 1.5T, Philips, Best, The Netherlands) [16]. For a safe approach avoiding vessels and sulci, a T1-weighted contrast-enhanced high-resolution 3D short gradient echo sequence was used. A few days before DBS implantation, DTI scanning was performed on a 3.0-tesla scanner (Achieva 3.0T, Philips, Best, The Netherlands) with the benefit of substantially higher signal-to-noise ratio compared to $1.5 \mathrm{~T}$ [17]. Acquisition parameters were as follows: echo time $70 \mathrm{~ms}$, sequence repetition time $6,495 \mathrm{~ms}$, matrix size $112 \times 110$, fieldof-view $224 \times 224 \mathrm{~mm}^{2}$ and slice thickness $2 \mathrm{~mm}$. Diffusion sensitizing gradients were applied in 32 directions according to a protocol provided by the manufacturer with a b-factor of $800 \mathrm{~s} / \mathrm{mm}^{2}$. To reduce geometric distortions, parallel imaging with the sensitivity encoding technique was used (SENSE) with a factor 2 .

\section{DTI Postprocessing, Tractography}

First, DTI was corrected by an affine registration for head motion and geometric distortions due to eddy currents [18]. The image without diffusion sensitizing gradients was used as a reference image. After correction the diffusion tensor was estimated for every voxel, and a deterministic tractography analysis was done with fiber assignment by continuous tracking (FACT) algorithm [13]. A multi-row approach was used for estimating the STC from the posterolateral medulla to the sensory cortex according to Hong et al. [14]. Stop criteria were a fractional anisotropy of less than 0.15 and a tracking angle of more than $60^{\circ}$. To correct for geometric distortion mainly due to susceptibility variations, a nonrigid free form deformation analysis was performed with the Image Registration Toolkit (ITK, Ixico Ltd., London, UK) [19]. The distortion field was estimated from the image without diffusion sensitizing gradients and a conventional turbo-spin-echo T2-weighted image. The control point spacing of the distortion field was set to $10 \mathrm{~mm}$, and a b-spline interpolation between the control points was used. The estimated distortion field, a displacement vector field, was applied to the reconstructed fiber tract. After geometric distortion correction, the tract was integrated into the treatment planning. 


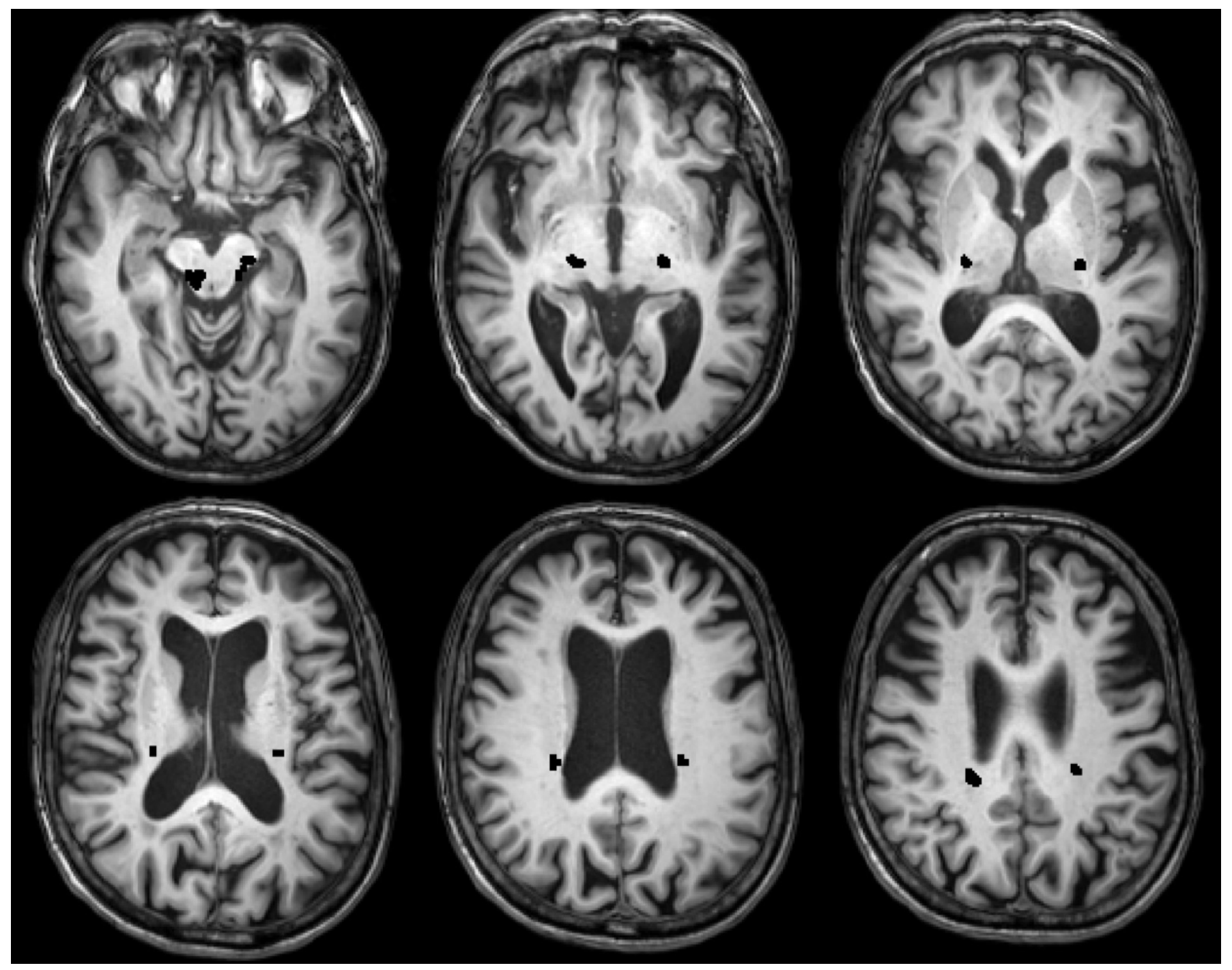

Fig. 1. Projection of the STC tract (black spots) in T1-weighted axial MRIs.

\section{Treatment Planning}

Treatment planning was done using stereotactic neurosurgery guidance software (STP3, Leibinger, Freiburg, Germany). The CT coordinates were transformed to stereotactic coordinates with the $\mathrm{V}$-shaped localizers. The MR image coordinates were transformed to stereotactic CT coordinates by landmark transformation [16].

The anterior and posterior commissure line (AC-PC line) was delineated on the T1-weighted images. As a first step, the target point was indirectly defined. The PLIC was divided into 3 parts and the posterior third part at the height of the AC-PC line was defined as the preliminary target point. Then, in a second step, the constructed STC was projected onto the T1-weighted images and the target point was slightly corrected (less than $2 \mathrm{~mm}$ ) to the center of the projected fiber tract at the height of the AC-PC line. With the help of the projected tractography onto the T1-weighted image (fig. 1), a target region was chosen in such way that the DBS electrode covered at a length of over $20 \mathrm{~mm}$ the sensory fiber from the target in the direction of the entry point. The extrapolation of a straight line to the skull defined the trajectory for the electrode implantation and resulted in an entry point at the postcentral gyrus. To avoid vessels and sulci the entry point was shifted gradually with negligible variations in the target region.

\section{Neurosurgical Procedure}

As usual, a temporary macro-electrode (TCB 013, FischerLeibinger, Freiburg, Germany) was inserted with a RiechertMundinger aiming bow. Macro-stimulation started at the dorsal part of the target trajectory of the thalamocortical tract in the PLIC approximately $20 \mathrm{~mm}$ above the target point. Test stimulation was done in a step length of $5 \mathrm{~mm}$, because the permanent DBS lead electrode model 3391 from Medtronic, which was planned for permanent implantation, yields a long range of $24 \mathrm{~mm}$ for stimulation 

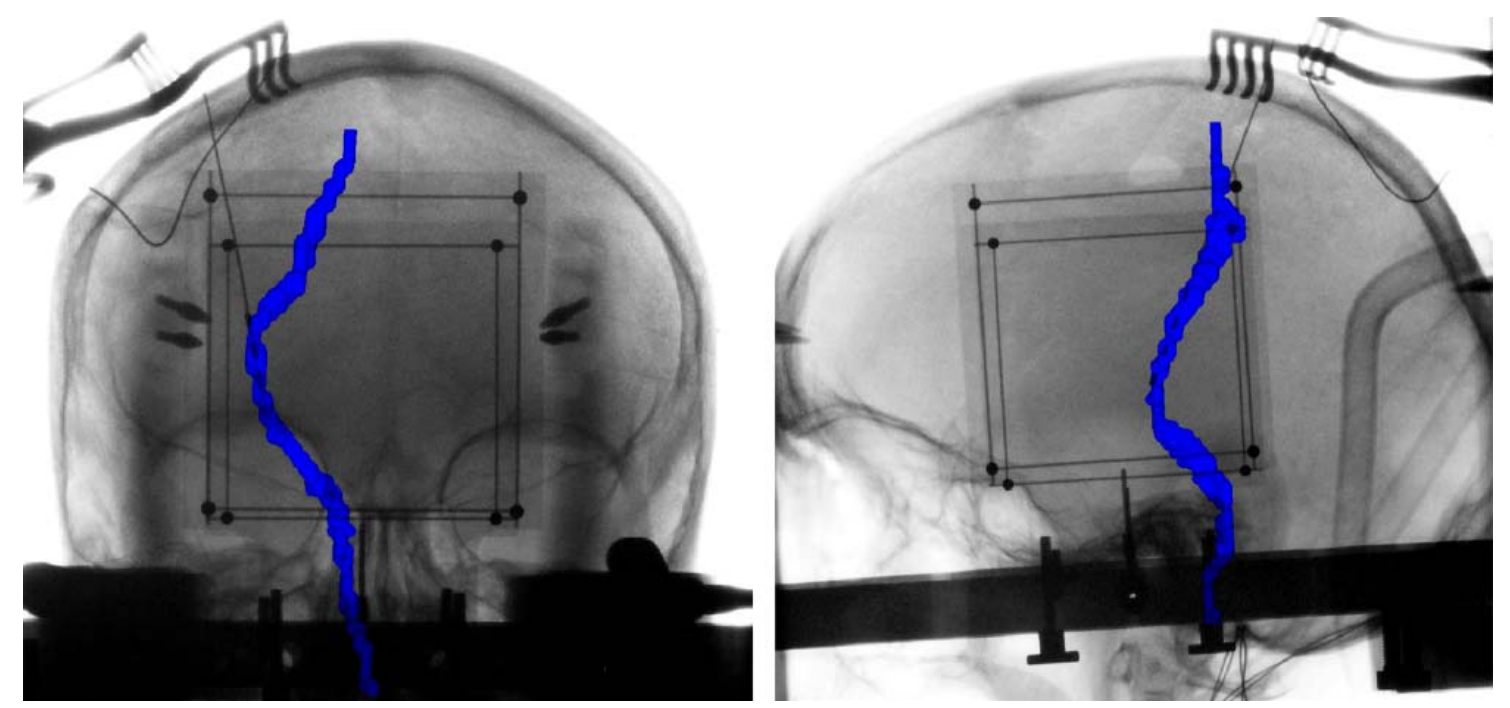

Fig. 2. Projection of STC tract (blue) in the intraoperative frontal (left) and lateral (right) X-ray images.

with an electrode contact length of $3 \mathrm{~mm}$ and a contact spacing of $4 \mathrm{~mm}$. Test stimulation parameters for the macro-electrode were set to a frequency of $130 \mathrm{~Hz}$ and pulse duration of 20-60 $\mu \mathrm{s}$. The voltage was increased stepwise in 0.1 -volt steps and the patient was asked about typical sensory effects such as a prickling sensation and pain relief.

A permanent DBS lead (model 3391, Medtronic, USA) and a neurostimulator (Soletra or Activa RC, Medtronic) were implanted if patients reported pain relief greater than $50 \%$ on the visual analogue scale during intraoperative test stimulation.

\section{Results}

\section{General}

Diffusion tensor imaging was successfully performed in all patients and an anatomical fitting of the STC, comparable to other DTI studies $[14,15]$, could be reconstructed. None of the calculated tracts was interrupted by the lesion. Figure 1 shows representative axial MR images of the fourth patient. The sensory tract is projected onto the axial images as black spot at different levels.

Figure 2 shows the frontal and lateral intraoperative $\mathrm{X}$-ray images with the implanted DBS lead. The sensory tract was projected onto the intraoperative $\mathrm{X}$-ray images with custom-made software [16]. The images were used for verification of the correct DBS-lead implantation [16]. The DBS lead covered a long range of the sensory tract in the PLIC thus yielding a target trajectory for multicontact stimulation instead of one target point.

DTI-Guided Stimulation of Somatosensory Fibers
In all 4 patients, the projection of the tractography onto the anatomical MRI images showed a consistent pattern of its localization in the PLIC. The tractography resulted in a target point at the level of the AC-PC line with a range of 16.7-24.4 $\mathrm{mm}$ lateral to the midline and 4.5-5.9 posterior to the mid-commissural point.

With trajectory guided implantation a mean implantation length of $24 \pm 4 \mathrm{~mm}$ with sensory stimulation effects (i.e. a prickling sensation or tingling) caused by stimulation voltages below $0.5 \mathrm{~V}$ was possible. The stimulated body parts varied depending on the level of the electrode without a recognizable systematic pattern. In all patients, the sensory stimulation of the affected whole body site was possible with one or more electrode contacts.

Intraoperatively, all patients reported a satisfying pain relief. After that evaluation a consensus decision (patient and physicians) was made about the permanent implantation of an internal neural stimulator. In 1 patient, the permanent DBS lead was implanted into the extracapsular part of the thalamocortical tract because of greater pain relief compared to the intracapsular stimulation.

Table 2 shows pre- and postoperative VAS pain assessment. In 3 of 4 patients, a pain relief of more than $40 \%$ was documented after 12 months. In 1 of 4 patients, the intraoperative successful pain relief vanished during the first 3 months. In this patient, only minor pain relief (10\%) could be reached even with different stimulation paradigms. In 2 patients, a significant reduction of the pain medication could be achieved (in patient 1 a reduction of $50 \%$, in patient 3 a reduction of $100 \%$ ).

Stereotact Funct Neurosurg 2013;91:328-334 
Table 2. VAS pre- and postoperation and optimum stimulator settings

\begin{tabular}{llllll}
\hline $\begin{array}{l}\text { Patient } \\
\text { No. }\end{array}$ & VAS (preop.) & $\begin{array}{l}\text { VAS (postop.) } \\
3 \text { month }\end{array}$ & $\begin{array}{l}\text { VAS (postop.) } \\
6 \text { month }\end{array}$ & $\begin{array}{l}\text { VAS (postop.) } \\
12 \text { month }\end{array}$ & $\begin{array}{l}\text { Stimulator settings active contacts/ } \\
\text { voltage }(\mathrm{V}) / \text { pulse width }(\mu s) / \text { rate }(\text { Hz) }\end{array}$ \\
\hline 1 & 10 & 6 & 6 & 6 & $-0,-1,-2,+3 / 4.5 / 150 / 60$ \\
2 & 10 & 5 & 6 & 6 & $-0,+\mathrm{G} / 1.0 / 60 / 60$ \\
3 & 10 & $2-3$ & $2-3$ & $2-3$ & $-2,-3,+\mathrm{G} / 1.2 / 90 / 20$ \\
4 & 10 & 9 & 9 & 9 & $-0,-1,-2,+3 / 2.0 / 60 / 60$ \\
\hline
\end{tabular}

VAS $=$ Visual analogue scale

\section{Discussion}

Interaction between the stimulation fields of the DBS electrodes and targeted neural tracts is regarded as causal for successful DBS treatment [20]. Guiding DBS implantation by visualization of neural pathways should thus be advantageous for a precise targeting. Diffusion-weighted MRI has been introduced as a noninvasive and in vivo technique for the delineation of specific white matter tracts $[21,22]$. For stimulation planning in patients with thalamic pain we used diffusion imaging-based tractography for guiding the implantation of DBS leads in combination with conventional MRI. We have shown that with tractography imaging a long-distance stimulation of the somatosensory pathway at the height of the PLIC with multiple electrode contacts is possible.

\section{DTI and Tractography}

For construction of the fiber pathway, we modeled diffusion with a diffusion tensor and combined it with the FACT algorithm [13]. This is one of the most reliable approaches for tractography of the main white matter tracts like the somatosensory tract $[15,23]$. Requirements on data acquisition are low and fit well into the clinical workflow. Furthermore, we used a multi-ROI approach for reconstruction of the fibers which is almost operator-independent [23].

The chosen ROI approach by Hong et al. [14] seems to allow a reliable STC reconstruction in the PLIC in patients with thalamic pain. Reconstruction parts near the lesion are difficult to assess. Because of low-diffusion anisotropy we cannot exclude that any reconstruction in these areas may not represent the actual anatomy. We also have to point out, as seen in figure 2, that the most centrifugal projections of the fibers near the cortical layer are missing. This is inherent to our tracking approach which stops tracking at low anisotropy values to reduce false-positive reconstructions.
One way of neurosurgical target definition is based on contrast information at the level of the target. Depending on the target structure as well the size of the target this can be achieved with conventional imaging. Sometimes, DTIgenerated color maps from the local orientation of the eigenvector seem to be useful, for instance for the thalamus. For the PLIC both contrast-based methods are not feasible. At the level of the posterior limb of the internal capsule all fibers show nearly the same orientation whether they are part of the STC or not. This results in a homogenous and according to the typical coding blue depiction of the whole PLIC in DTI-generated color maps, i.e. in an image without any useful contrast information. Therefore, the fiber tract information which is based on seed points seems mandatory for a target generation within the PLIC. Interestingly, it turned out that the rule of thumb estimation for the preliminary target point at the height of the AC-PC line differed less than $2 \mathrm{~mm}$ from the tractography-based. But the target information at the level of the AC-PC line is only one part of the necessary information for a multielectrode approach, since for such a long-range target the directional information above this target at the level of the AC-PC line is vital. None of the other methods except for tractography can deliver this.

\section{Planning of the DBS Lead}

The reconstructed sensory fiber tract was projected onto the axial planning data. By extrapolation of a line through the first decimeter of the tract in the PLIC to the skull, the postcentral gyrus turned out as a proper entry point for the DBS lead resulting in a stimulation length of more than $20 \mathrm{~mm}$.

In our patients, an entire side of the body is affected by the pain. Therefore, a short distance from the thalamus is preferable for positioning of the DBS lead to include most of the fibers of the thalamocortical tract hereby avoiding a fanning out of the STC as seen in its extracapsular part. Apart from that, the desired effect should 
be maximized and side effects caused by stimulation of other tracts minimized by a central positioning of the electrodes within the tract under the main assumption that selective stimulation of the somatosensory tract is responsible for pain relief. Because of its tight bundling of the thalamocortical pathway at the point of departure from the thalamus and its further way between thalamus and lentiform nucleus, the PLIC seems anatomically ideal for targeting. PLIC stimulation should result in an unselective stimulation with respect to the somatotopy and a selective stimulation concerning the main tract anatomy. Placing DBS leads with several electrode contacts along this pathway increases the likelihood of effective stimulation either by different electrode contact locations for a mono-electrode stimulation paradigm or for multiple-electrode stimulation with the advantage of spatially or temporally fractionated application of electric fields.

\section{Intraoperative Stimulation Results}

Fiber tracking is prone to false-positive or false-negative pathway constructions because its calculation is based on an ill-posed mathematical problem. There exists no single solution of fiber structure modeling for the acquired diffusion data. Our intraoperative stimulation results with sensory stimulation show effects like prickling sensation or tingling with stimulation parameters below $0.5 \mathrm{~V}$ which indicate to certain degree a valid modeling. In our opinion, it is too far stretched to interpret positive intraoperative stimulation results as hard proof for a correct tract modeling and evidence for a perfect localization of the electrode within the sensory fibers. Furthermore, fiber disintegration such as Waller degeneration may need different stimulation levels to achieve a positive stimulation response.

However, we could stimulate the neural target tract over a very long distance with multiple electrode contacts resulting in an extensive stimulation area. In our opinion, this approach increases the likelihood of a successful stimulation result.

\section{Clinical Results}

Three of 4 patients experienced satisfying pain relief over a time length of at least one year. None demanded removal of the stimulation system. Our preliminary results are promising in comparison to other approaches like motor cortex stimulation where a satisfactory pain reduction was only reached in about half of the patients [24], especially in cases where the pain projects to the whole hemisphere. We were not able to explain why 1 pa- tient could not profit from the lead implantation. The lesion characteristics as well as the reconstruction of the STC tract did not differ from the other successful stimulated patients.

\section{Conclusion}

Our novel approach of integrating tractography data into DBS planning in thalamic pain treatment is technically feasible and allows long-distance placement of multiple electrode contacts along the thalamocortical tract at the level of the PLIC. In our first 4 patients, tingling at low stimulation levels indicates an anatomical validity of our tract modeling. Based on effects of over $40 \%$ pain reduction after 12 months for most of our patients our approach seems to have a promising clinical potential. By using the multiple electrode option in the future sophisticated stimulation paradigms may turn out as a distinctive advantage of the tractography approach over conventional single-point targeting. A larger number of cases in a prospective study are necessary to validate our results.

\section{Acknowledgments}

We received no financial support in conjunction with our work on this submission. The Image Registration Toolkit was used under license from Ixico Ltd.

References

Stereotact Funct Neurosurg 2013;91:328-334 333
1 Leijon G, Bovie J, Johansson I: Central poststroke pain: neurological symptoms and pain characteristics. Pain 1989;36:13-25.

2 Boivie J, Leijon G, Johansson I: Central poststroke pain - a study of the mechanisms through analyses of the sensory abnormalities. Pain 1989;37:173-185.

3 Hong JH, Choi BY, Chang CH, Kim SH, Jung YJ, Lee DG, Kwon YH, Jang SH: The prevalence of central poststroke pain according to the integrity of the spino-thalamo-cortical pathway. Eur Neurol 2012;67:12-17.

4 Adams JE, Hosobuchi Y, Fields HL: Stimulation of internal capsule for relief of chronic pain. J Neurosurg 1974;41:740-744.

5 Franzini A, Cordella R, Nazzi V, Broggi G: Long-term chronic stimulation of internal capsule in poststroke pain and spasticity. Case report, long-term results and review of the literature. Stereotact Funct Neurosurg 2008;86: 179-183.

6 Hosobuchi Y, Adams JE, Rutkin B: Chronic thalamic and internal capsule stimulation for the control of central pain. Surg Neurol 1975; 4:91-92. 
7 Owen SL, Green AL, Stein JF, Aziz TZ: Deep brain stimulation for the alleviation of poststroke neuropathic pain. Pain 2006;120:202206.

8 Tsubokawa T, Katayama Y, Yamamoto T, Hirayama T, Koyama S: Chronic motor cortex stimulation in patients with thalamic pain. J Neurosurg 1993;78:393-401.

9 Nguyen JP, Lefaucheur JP, Decq P, Uchiyama $T$, Carpentier A, Fontaine D, Brugières $\mathrm{P}$, Pollin B, Fève A, Rostaing S, Cesaro P, Keravel Y: Chronic motor cortex stimulation in the treatment of central and neuropathic pain. Correlations between clinical, electrophysiological and anatomical data. Pain 1999;82:245-251.

10 Basser PJ, Mattiello J, LeBihan D: Estimation of the effective self-diffusion tensor from the NMR spin echo. J Magn Reson B 1994;103: 247-254.

11 Moseley ME, Cohen Y, Kucharczyk J: Diffusion-weighted MR imaging of anisotropic water diffusion in cat central nervous system. Radiology 1990;176:439-445.

12 Conturo TE, Lori NF, Cull TS, et al: Tracking neuronal fiber pathways in the living human brain. Proc Natl Acad Sci USA 1999;96:1042210427.
13 Mori S, Crain BJ, Chacko VP, van Zijl PC: Three-dimensional tracking of axonal projections in the brain by magnetic resonance imaging. Ann Neurol 1999;45:265-269.

14 Hong JH, Son SM, Jang SH: Identification of spinothalamic tract and its related thalamocortical fibers in human brain. Neurosci Lett 2010;468:102-105.

15 Yamada K, Akazawa K, Yuen S, et al: MR imaging of ventral thalamic nuclei. AJNR 2010; 31:732-735.

16 Hunsche S, Sauner D, Maarouf M, Lackner K, Sturm V, Treuer H: Combined X-ray and magnetic resonance imaging facility: application to image-guided stereotactic and functional neurosurgery. Neurosurgery 2007;60: 352-360.

17 Hunsche S, Moseley ME, Stoeter P, Hedehus M: Diffusion-tensor MR imaging at 1.5 and 3.0 T: initial observations. Radiology 2001; 221:550-556

18 Smith SM, Jenkinson M, Woolrich MW: Advances in functional and structural MR image analysis and implementation as FSL. Neuroimage 2004;23:208-219.

19 Rueckert D, Sonoda LI, Hayes C, Hill DLG, Leach MO, Hawkes DJ: Non-rigid registration using free-form deformations: application to breast MR images. IEEE Trans Med Imaging 1999;18:712-721.
20 Gradinaru V, Mogri M, Thompson K, Henderson J, Deisseroth K: Optical deconstruction of parkinsonian neural circuitry. Science 2009;324:354-359.

21 Jones DK: Studying connections in the living human brain with diffusion MRI. Cortex 2008;44:936-952.

22 Fillard P, Descoteaux M, Goh A, Gouttard S, Jeurissen B, Malcolm J, Ramirez-Manzanares A, Reisert M, Sakaie K, Tensaouti F, Yo T, Mangin JF, Poupon C: Quantitative evaluation of 10 tractography algorithms on a realistic diffusion MR phantom. Neuroimage 2011;56:220-234.

23 Wakana S, Caprihan A, Panzenboeck MM, Fallon JH, Perry M, Gollub RL, Hua K, Zhang J, Jiang H, Dubey P, Blitz A, van Zijl P, Mori $S$ : Reproducibility of quantitative tractography methods applied to cerebral white matter. Neuroimage 2007;36:630-644.

24 Katayama Y, Yamamoto T, Kobayashi K, Kasai M, Oshima H, Fukaya C: Motor cortex stimulation for post-stroke pain: comparison of spinal cord and thalamic stimulation. Stereotact Funct Neurosurg 2001;77:183-186. 